\title{
PERFIL DE PACIENTES COM INFARTO AGUDO DO MIOCÁRDIO NA PERSPECTIVA DO MODELO DE "CAMPO DE SAÚDE"
}

\author{
InFARTED PATIENT'S PROFILE CONCERNING THE "HEALTH AREA" MODEL \\ RASGOS DE PACIENTES CON INFARTO AGUDO DE MIOCARDIO EN LA' \\ PERSPECTINA DEL MODELO DE "CAMPO DE SALUD"
}

\section{Rosana Aparecida Spadoti Dantas ${ }^{2}$ Olga Maimoni Aguilar}

\begin{abstract}
RESUMO: Estudo realizado com o objetivo de conhecer o perfil dos infartados atendidos no Hospital das Clínicas de Ribeirão Preto, entre maio e novembro de 1994. Os dados foram coletados através de entrevista com os pacientes e consulta ao prontuário médico. Os resultados obtidos e analisados, segundo o Modelo de "Campo de Saúde", foram: a) biologia humana - 66,7\% do sexo masculino; $73,2 \%$ na faixa 50 - 80 anos; $55,5 \%$ hipertensos; $24,4 \%$ dislipidêmicos; $20 \%$ diabéticos; $51,1 \%$ com história familiar positiva para hipertensão; $26,6 \%$ para infarto e 24,4\% para acidente vascular cerebral; b) caracterização sócio-econômica: $71,1 \%$ com renda mensal menor que 6 salários-mínimos; $82,2 \%$ analfabetos ou com primeiro grau incompleto; $47 \%$ economicamente ativos e $68,8 \%$ casados;c) estilo de vida: $88,8 \%$ sedentários; $55,5 \%$ tabagistas e $55,4 \%$ referiram estresse diário; d) atenção à saúde: $68,8 \%$ acompanhados em serviços de saúde; $75,5 \%$ conheciam o diagnóstico atual e $62,2 \%$ solicitavam informações sobre fisiopatologia, prognóstico e reabilitação. A presença de 2 ou 3 fatores de risco para doença isquêmica cardíaca foi verificada em $62,2 \%$ dos pacientes. Em relação ao modelo utilizado constatou-se, em seus quatro elementos, a presença de fatores de risco para o infarto.
\end{abstract}

PALAVRAS CHAVE: Infarto agudo do miocárdio, Fatores de risco, Modelo de "Campo de Saúde" , Estilo de vida.

\begin{abstract}
The present study aimed at finding out the profile of patients with acute myocardial infarction into University Hospital at Ribeirão Preto, from May to November, 1994. Data were collected through interviews with the patients and analysis of the medical reports. The results were analysed according to the "Health Field Model" and were the following: a) human biology: $66,7 \%$ were men; $73,2 \%$ of them were from 50 to 80 years old; $55,5 \%$ were hypertensive; $24,4 \%$ with dyslipidemia; $20 \%$ had diabetes; $51,1 \%$ had a positive family history of hypertension, $26,6 \%$ of had infarction and $24,4 \%$ had cerebral stroke; b) socioeconomic characterization: $71,1 \%$ had a monthly income lower than 6 minimum salaries; $82,2 \%$ illiterate or with incomplete primary school; $47 \%$ were economically active and $68,8 \%$ were married; c) life style: $88,8 \%$ lived a sedentary life; $55,5 \%$ smoked and $55,4 \%$ referred to daily stress; d) attention to health: $68,8 \%$ were being treated in health services: $75,5 \%$ knew about their diagnostic and $62,2 \%$ asked about informations on physiopathology, prognostic and rehabilitation. The presence of 2 or 3 risk factors to cardiac diseases was verified in $62,2 \%$ of the patients. Regarding the model, authors found the presence of risk factors to infarction in its four elements.
\end{abstract}

KEY WORDS: myocardial infarct, "Health Field" Model, risk factors, life style.

\footnotetext{
${ }^{1}$ Resumo de Dissertação de Mestrado apresentada à Escola de Enfermagem de Ribeirão Preto, Universidade de São Paulo (EERP, USP).

2 Enfermeira, aluna do Programa Interunidades de Doutorado da EERP, USP.

${ }^{3}$ Enfermeira, Professora Doutora junto ao Departamento de Enfermagem Geral e Especializada da EERP, USP.
} 
RESUMEN: Estudio realizado com el objetivo de conocer los rasgos de los infartados atendidos en el Hospital de Clínicas de Ribeirão Preto, entre mayo y noviembre de 1994.Los datos fueron recoletados a través de entrevistas con los pacientes y consultas a prontuarios médicos. Los resultados, analisados siguiendo el modelo de "Campo de la salud", fueron los seguintes:a) biologia humana- $66,7 \%$ de sexo masculino; $73,2 \%$ entre 50 y 80 año; $55,5 \%$ hipertnsos; $24,4 \%$ dislipidémicos; $20 \%$ diabéticos; $51,1 \%$ com historia familiar positiva para hipertensión, 26,6\% para infaro y $24,4 \%$ para accidente vascular cerebral:b)características socio-económicas:71,1\% con ingresos mensuales menores que 6 salarios mínimos; $82,2 \%$ analfabetos o con la escuela prima incompleta, $47 \%$ económicamente activos y $68,8 \%$ casados;c)estilo de vida:88,8\% sedentarios; $55,5 \%$ fumadores y $55,4 \%$ refiriendo stress diario; d)atención a la salud: $68,8 \%$ siendo tratados en servicios de salud; $75,5 \%$ conocian el diagnóstico actual y $62,2 \%$ solicitaron informaciones sobre fisiopatología, pronóstico y rehabilitación. La presencia de 2 o 3 factores de riesgo para la enfermedad de isquemia cardíaca fue verificada en $62,2 \%$ de los pacientes. En relación al modelo utilizado, se constató la presencia de riesgo para infarto en sus cuatro elementos.

PALABRAS CLAVE: infarto de miocardio, modelo "Campo de la salud", factores de riesgo

\section{INTRODUÇÃO}

Dentre as doenças crônico-degenerativas, as doenças cardiovasculares ocupam a liderança como causa de morte em todo o mundo e, no Brasil, já são responsáveis por, pelo menos, 300 mil óbitos anuais, além de terem o tratamento mais dispendioso do que qualquer outra síndrome mórbida e de representarem os maiores custos da previdência com licenças e aposentadorias antecipadas. (BANCO MUNDIAL, 1991; BRASIL, 1993).

Estudos têm demonstrado que entre as doenças cardiovasculares está sendo percebida uma diminuição na mortalidade decorrente de doença isquêmica cardíaca e doença cérebro-vascular, nos últimos anos. A queda da mortalidade da doença isquêmica, no Brasil, começou a ser observada na década de 70. Infelizmente, tal declínio não retira a doença cardiovascular da posição de líder, sendo um dos principais componentes de morbi-mortalidade. (Rego, 1990).

Estudo realizado nas regiões metropolitanas brasileiras, entre 1979 e 1989, por Lolio et al. (1995), mostrou que os coeficientes de mortalidade por doença isquêmica cardíaca apresentaram queda em Belém e São Paulo, para ambos os sexos, e em Belo Horizonte para o sexo feminino. Tais coeficientes permaneceram estáveis em Salvador, Curitiba e Porto Alegre, enquanto apresentaram ascensão em Recife e Rio de Janeiro.

Na cidade de Ribeirão Preto, estado de São Paulo, um levantamento mostrou que, no ano de 1993, foram internados no Hospital das Clínicas, 351 pacientes com doença isquêmica cardíaca, exceto casos de angina, sendo que destes, 215 $(61,2 \%)$ tinham diagnóstico de infarto agudo do miocárdio. Em 1994, apesar do número de internações ter sido praticamente o mesmo, com 350 pacientes, houve diminuição do número de infarto para 178 (50,8\%). (SÃO PAULO, 1996).

Através da nossa vivência no atendimento de infartados, no referido hospital, pudemos observar que estas pessoas têm pouco ou nenhum conhecimento a respeito das posssíveis causas, do prognóstico e do comprometimento de suas vidas em decorrência da doença.

Ao identificarmos essa lacuna no que diz respeito à orientação dos pacientes 
que sofreram infarto agudo do miocárdio e que são atendidos nesse hospital, sentimos a necessidade de investirmos nesta área, levando tais pessoas a compreenderem mais claramente a situação em que se encontram e fornecer-lhes condições para participar mais ativamente de suas reabilitações. Neste sentido, nos propusemos no presente estudo, a conhecer o perfil dos indivíduos infartados atendidos no referido serviço, obtendo desta forma, informações que possam subsidiar a elaboração de um programa de reabilitação para estes pacientes.

\section{REFERENCIAL TEÓRICO: O MODELO DE "CAMPO DE SAÚDE"}

Buscando subsídios para a elaboração do nosso estudo, escolhemos o modelo conceitual denominado de "Campo de Saúde", proposto por Marc Lalonde (1974). Tal modelo foi escolhido por possibilitar-nos uma análise mais ampla dos problemas da área da saúde, no caso, a incidência de infarto agudo do miocárdio e a reabilitação dos doentes atingidos. Tal modelo considera, além dos aspectos biológicos, outros fatores que podem contribuir para a determinação desta doença multifatorial.

O documento publicado pelo autor e intitulado Uma nova perspectiva sobre a sailde dos canadenses (Lalonde, 1974), traz sua preocupação com relação à ausência de uma estrutura conceitual para analisar a área da saúde. Através do exame das causas e fatores básicos de doenças e mortes no Canadá e do levantamento da participação destes elementos no estabelecimento do nível de saúde da população canadense, foram estabelecidos quatro elementos principais que compõem o modelo de "Campo de Saúde", são eles: biologia humana, meio ambiente, estilo de vida e organização dos serviços de saúde.Tais elementos são assim definidos pelo autor:

biologia lummana: inclui todos aqueles aspectos da saúde, física e mental, os quais pertencem ao corpo humano e à constituição orgânica do indivíduo. Relaciona-se à herança genética, ao processo de maturidade e de envelhecimento e aos muitos sistemas orgânicos. Sendo um organismo complexo, o corpo humano tem implicações numerosas, variadas e sérias na saúde individual, contribuindo para todos os tipos de doenças e mortalidade;

meio ambiente: inclui todos aqueles aspectos relacionados à saúde e que estão externos ao corpo humano, quer pertençam ao ambiente físico, quer pertençam ao ambiente social que envolve os indivíduos, e sobre os quais eles têm pouco ou nenhum controle como, por exemplo, o controle da qualidade da água e do ar, no ambiente físico, e o controle das mudanças aceleradas ocorridas no ambiente social;

estilo de vida: consiste no conjunto de decisões tomadas pelo indivíduo, sobre as quais ele possui maior ou menor controle, e que afetam a sua saúde. Do ponto de vista da saúde, decisões e hábitos pessoais ruins criam os chamados riscos autocriados. Quando esses riscos resultam em doenças e mortes pode-se dizer que o "estilo de vida" de suas vítimas contribuiu para ou causou tais ocorrências;

organização dos serviços de saúde: refere-se à qualidade, quantidade, administração, natureza e relações de pessoas e recursos no oferecimento do cuidado de 
saúde. Inclui médicos, enfermeiros, hospitais, farmácias, serviços de saúde públicos e privados, ambulatórios e serviços odontológicos entre outros. É comumente definido como sistema de atenção à saúde.

Neste modelo, os quatro elementos acima tem o mesmo valor na determinação da saúde do indivíduo e precisam estar em equilíbrio para que tal condição se estabeleça. (Lalonde, 1974).

Com relação ao estilo de vida, o autor acredita que a presença de hábitos destrutivos, no dia a dia, dos indivíduos acarreta vários tipos de enfermidades como, por exemplo, as doenças cardiovasculares. Tais hábitos estão presentes no uso de drogas (ingestão excessiva de bebidas alcoólicas, fumo e abuso de medicamentos) e na relação dieta-exercícios (super alimentação; alta ingestão de gorduras e carboidratos; falta de exercícios físicos e de recreação e relaxamento das tensões diárias).

Para um melhor embasamento dos fatores de risco específicos para as doenças cardiovasculares, principalmente para a ocorrência do infarto do miocárdio, buscamos na literatura os fatores de risco determinantes para estas doenças.

O termo "fator de risco" relacionado ao desenvolvimento das doenças cardiovasculares foi usado pela primeira vez no estudo de Framinghan, realizado em uma pequena cidade perto de Boston (EUA) e iniciado no final da década de 40. Através do mesmo foi possível estabelecer quais eram as variáveis a serem pesquisadas na determinação do risco individual de doença cardiovascular. (Guimarães, 1992, Kannel, 1976, Mancilha-Carvalho, 1992).

Os fatores de risco que predispõem as pessoas a tais doenças dividem-se em quatro grupos: hábitos do estilo de vida (ingestão aumentada de gorduras saturadas, colesterol e calorias; sedentarismo; tabagismo; padrão de comportamento tipo A e ganho irrestrito de peso); traços individuais aterogênicos (hipertensão arterial sistêmica; dislipidemias; intolerância à glicose e resistência à insulina); susceptividade inata (predisposição genética para dislipidemias, hipertensão e diabetes e história familiar positiva de doença cardiovascular prematura) e parâmetros preditores aterogênicos ativos (leucocitose e fibrinogênio elevado). Atualmente, os principais fatores de risco são: dislipidemias, hipertensão arterial sistêmica, tabagismo e intolerância à glicose (Kannel, 1995).

Os fatores de risco também podem ser classificados de acordo com as suas possibilidades de modificação, quer seja através de mudanças de comportamentos, quer seja por outros tipos de tratamento. No grupo do modificáveis, temos a hipertensão arterial sistêmica, a intolerância à glicose e Diabetes mellitus não insulino-depeńdente, sedentarismo, alcoolismo e uso de anticoncepcionais hormonais. Os não modificáveis são: raça, sexo e idade, história familiar positiva e ocorrência da menopausa. (Kannel, 1995, Mancilha-Carvalho, 1992).

\section{OBJETIVOS}

Os objetivos determinados para o presente estudo são:

caracterizar os indivíduos acometidos por primeiro infarto agudo do miocárdio 
quanto aos seguintes aspectos: biologia do indivíduo, meio ambiente, estilo de vida e organização dos serviços de saúde, de acordo com o modelo conceitual

- de "Campo de Saúde", proposto por Lalonde, e

levantar conhecimentos e dúvidas dos pacientes relativos ao infarto.

\section{METODOLOGIA}

O estudo foi realizado junto à Divisão de Cardiologia do Hospital das Clínicas de Ribeirão Preto e abordou os pacientes internados na Unidade Coronariana do referido hospital, no perído de 1 de maio a 30 de novembro de 1994.

Foram entrevistados 45 pacientes que atenderam aos seguintes requisitos: ter sofrido primeiro episódio de infarto agudo do miocárdio (confirmado através de história clínica, traçado eletrocardiográfico e dosagem enzimática), estar em condições físicas e psicológicas de participar da entrevista ou ter um membro da familiar para responder às questões e concordar em participar da pesquisa.

A coleta de dados foi feita através de entrevista semi-estruturada e consulta ao prontuário médico dos pacientes. Para padronizar e facilitar a coleta dos dados foi elaborado um roteiro, dividido em grupos de dados específicos para cada elemento que compõe o modelo de "Campo de Saúde", ou seja, informações referentes à biologia humana, meio ambiente, estilo de vida e organização dos serviços de saúde. A escolha das informações levantadas e pertinentes a cada elemento do modelo foi feita mediante a definição proposta por Lalonde (1974), e englobando os fatores de risco para doenças cardiovasculares encontrados na literatura consultada. A elaboração do roteiro foi feita em parceria com Colombo (1995), a qual utilizou-o em seu estudo sobre o estilo de vida e fatores de risco coronarianos, realizado em Campinas, no ano de 1994.

As entrevistas foram realizadas no período compreendido entre 48 horas após a internação até o momento da alta hospitalar. Os dados foram coletados em impresso próprio, utilizado para cada um dos entrevistados e contendo o roteiro da entrevista. Do prontuário médico foram levantados dados referentes à confirmação do diagnóstico, data de ocorrência do infarto e internação na Unidade Coronariana: peso, altura e história clínica do paciente.

Realizada a coleta de dados, procedemos à eleboração e classificação das respostas de forma sistemática, permitindo uma análise dos mesmos. Os dados sofreram uma análise descritiva e percentual e os resultados possibilitaram a definição do perfil dos indivíduos infartados, relacionado aos elementos do Modelo de "Campo de Saúde" com a presença de fatores de risco coronarianos.

\section{RESULTADOS E DISCUSSÃO}

Conforme referencial teórico utilizado e com base no instrumento proposto para a coleta de dados, apresentamos os resultados agrupados nos seguintes ítens:

\subsection{DADOS DA BIOLOGIA DO INDIVÍDUO}

Entre os 45 pacientes entrevistados, a maior incidência de infarto agudo do miocárdio ocorreu nos indivíduos do sexo masculino, na proporção de $2 / 1$. A 
literatura, ao discutir o papel do fator sexo no desenvolvimento da doença isquêmica cardíaca, comumente o faz relacionando-o com a idade. Tal fato é decorrente das características peculiares a cada sexo e que fazem homens e mulheres envelhecerem de maneiras distintas (Aburdene, 1994; Kannel, 1987, Mancilha-Carvalho, 1992, Underhill; Stephen, 1984).

Observamos que, com relação à idade, a maior concentração de pacientes está na faixa etária dos 50 -70, sendo seguidos por aqueles com idade entre 70 e 80 anos. Tal resultado confirma que o infarto atinge, predominantemente, os indivíduos de meia idade e idosos (15). Ao compararmos as incidências entre os dois sexos constatamos que a maior diferença entre elas ocorreu na faixa dos 30-40 anos, 5 homens/1 mulher. Com o avançar da idade esta diferença diminuiu, porém, em todas as faixas etárias, a incidência no sexo masculino foi maior (Tabela 1).

TABELA 1: Distribuição dos 45 pacientes infartados, segundo o sexo e a faixa etária.

\begin{tabular}{|c|c|c|c|c|c|c|}
\hline \multirow[t]{2}{*}{ Faixa etária } & \multicolumn{2}{|c|}{ Homens } & \multicolumn{2}{|c|}{ Mulheres } & \multicolumn{2}{|l|}{ Total } \\
\hline & $n$ & $\%$ & $n$ & $\%$ & $n$ & $\%$ \\
\hline $30 \mid-40$ & 4 & 8,9 & 1 & 2,2 & 5 & 11,1 \\
\hline $40 \mid-50$ & 4 & 8,9 & 2 & 4,4 & 6 & 13,2 \\
\hline $50 \mid-60$ & 7 & 15,5 & 5 & 11,1 & 12 & 26,6 \\
\hline $60 \mid-70$ & 8 & 17,8 & 3 & 6,6 & 11 & 24,2 \\
\hline $70 \mid-80$ & 7 & 15,5 & 3 & 6,6 & 10 & 22,2 \\
\hline $801-$ & - & - & 1 & 2,2 & 2 & 2,2 \\
\hline Total & 30 & 66,7 & 15 & 33,3 & 45 & 100,0 \\
\hline
\end{tabular}

O sexo masculino é considerado fator de risco para doença isquêmica cardíaca, principalmente, para os homens com idade inferior a 50 anos. Até essa idade, o homem tem um risco de sofrer um infarto cerca de três vezes maior que a mulher da mesma idade. Após os 50 anos a diferença na incidência da doença entre os dois sexos diminui e as mulheres, após a menopausa, tornam-se tão vulneráveis ao infarto quanto os homens da mesma faixa etária. A vantagem da mulher sobre o homem, antes da menopausa, parece estar relacionada a alguns mecanismos da fisiologia reprodutiva, responsáveis por uma menor tendência trombolítica e uma proteção hormonal. (Johansson; Vedin; Wilhelmsson, 1983, Kannel, 1987).

A obesidade foi outro aspecto investigado por ser considerada como fator de risco ou agravamento para várias doenças, entre elas a hipertensão arterial, hipercolesterolemia e diabetes mellitus (BRASIL, 1993, Kannel, 1987, MancilhaCarvalho, 1992).

A distribuição dos 45 pacientes, segundo o índice de massa corporal (valor obtido através da divisão do peso do indivíduo, em quilos, pelo quadrado da sua altura, em metros) (INTERNATIONAL LIPID INFORMATION BUREAU (ILIB) LATINO AMERICA, 1994), mostra que a maioria dos entrevistados, $67,8 \%$, não era obesa, encontrando-se nas categorias de peso normal (IMC entre $20-25 \mathrm{~kg} / \mathrm{m} 2$ ) e sobrepeso (IMC entre $25-30 \mathrm{Kg} / \mathrm{m} 2)$. Assim, o fator obesidade não foi freqüente no grupo estudado. 
Outro ítem levantado foi a presença de história familiar positiva para doenças cardiovasculares entre os participantes do estudo. Verificamos que $51,1 \%$ dos pacientes tinham antecedentes familiares para hipertensão arterial; $28 \%$ para infarto agudo do miocárdio; $24 \%$ para acidente vascular cerebral; 22,2\% para dislipidemias, $20 \%$ para morte súbita e $16 \%$ para diabetes.

A história familiar positiva para as doenças acima mencionadas, entre parentes consangüíneos, é um fator de risco importante na determinação da ocorrência e prognóstico de doença isquêmica cardíaca, especialmente quando associada a outros fatores de risco. (Gianini, 1994, Guimarães, 1992, Anderhill; Stephen, 1984).

O último dado levantado referente à biologia do indivíduo foi a história clínica do paciente, buscando informações referentes à presença de doenças anteriores, como hipertensão arterial, dislipidemias e diabetes, entre outras, as quais são consideradas fatores de risco para o desenvolvimento do infarto agudo do miocárdio.

A hipertensão arterial sistêmica foi a mais prevalente, como antecedente pessoal, no grupo estudado, atingindo $55,5 \%$ dos entrevistados. No estudo de Framinghan a hipertensão dobrou o risco de doença isquêmica cardíaca. (Kannel, 1976, Kannel, 1987).

Outros antecedentes pessoais levantados foram: dislipidemias e doença vascular periférica, ambas atingindo $24,4 \%$ dos participantes; diabetes mellitus, $20 \%$ e acidente vascular cerebral, $8,9 \%$. Acreditamos que tais valores possam estar subestimados, principalmente no que se refere às dislipidemias, uma vez que $26,6 \%$ dos entrevistados referiram não saber a presença de alterações lipídicas em seus organismos. Tal desconhecimento é preocupante uma vez que a hipercolesterolemia, dentre as dislipidemias, é tida como um dos quatro principais fatores de risco para doença isquêmica cardíaca. (Kannel, 1995).

\subsection{DADOS DO MEIO AMBIENTE}

Através desses dados, buscamos informações que caracterizassem as condições sócio-econômicas dos 45 infartados, levando-se em consideração a ocupação, grau de instrução, renda familiar, número de filhos, estado civil e procedência dos mesmos.

Os resultados obtidos indicaram que o nível de instrução dos pacientes era precário, sendo a maioria composta por indivíduos analfabetos $(28,9 \%)$ e com primeiro grau incompleto (53,3\%). Tais dados são compatíveis com as peculiaridades de uma clientela atendida em um hospital-escola, como é o caso do Hospital das Clínicas de Ribeirão Preto, onde a maior parte dos pacientes atendidos não possuem condições sócio-econômicas para custearem seus gastos com a saúde.

Pesquisas apontam para a maior presença de um ou mais fatores de risco entre aqueles indivíduos com menor número de anos de estudo e que, mudanças no estilo de vida ficam mais evidentes naqueles com maior escolaridade, levando a 
crer que há uma associação inversa entre estrato social, nível educacional e doença cardíaca isquêmica, entre outras doenças cardiovasculares. (BANCO MUNDIAL, 1991, Bittar, 1992).

Quanto à ocupação, certos estudos têm demonstrado que a doença isquêmica cardíaca está entre os principais grupos de doenças e acidentes relacionados com o trabalho. (Bittar, 1992, Paffenbarger; Halle, 1975).

Utilizamos dois tipos de classificação para analisar as atividades profissionais dos entrevistados: a "Classificação Brasileira de Ocupações para o subsistema de informações sobre mortalidade" (BRASIL, 1987), do Ministério da Saúde e a classificação das ocupações segundo o esforço físico exigido (leve ou pesado) e a promoção de alterações no biorritmo do indivíduo (trabalhos em turnos).

TABELA 2: Distribuição dos 45 pacientes infartados, segundo à Classificação Brasileira de Ocupações (M.S.) ${ }^{\star}$, o esforço físico exigido e alteração do biorritmo.

\begin{tabular}{|c|c|c|c|c|c|c|c|c|}
\hline \multirow{2}{*}{$\begin{array}{l}\text { OCUPAÇÃO } \\
\text { (Código) }\end{array}$} & \multicolumn{2}{|c|}{ LEVE } & \multicolumn{4}{|c|}{$\begin{array}{l}\text { PESADA ALT. D } \\
\text { BIORRITMO }\end{array}$} & \multicolumn{2}{|c|}{ TOTAL } \\
\hline & $\mathrm{n}$ & $(\%)$ & $n$ & $(\%)$ & $\mathrm{n}$ & $(\%)$ & $\mathrm{n}$ & $(\%)$ \\
\hline aposentado (007) & 14 & 31,1 & - & - & - & - & 14 & 31,1 \\
\hline dona de casa (008) & 10 & 22,2 & - & - & - & - & 10 & 22,2 \\
\hline pessoal de enfermagem $((072)$ & 1 & 2,2 & - & - & - & - & 1 & 2,2 \\
\hline empreiteiro de obras (234) & 1 & 2,2 & - & - & - & - & 1 & 2,2 \\
\hline bancário (390) & 1 & 2,2 & - & - & - & - & 1 & 2,2 \\
\hline comerciante (410) & 2 & 4,4 & - & - & - & - & 2 & 4,4 \\
\hline jardineiro (540) & - & - & 1 & 2,2 & - & - & 1 & 2,2 \\
\hline guarda noturno (583) & - & - & - & - & 3 & 6,7 & 3 & 6,7 \\
\hline lavador de carro (599) & - & - & 1 & 2,2 & - & - & 1 & 2,2 \\
\hline lavrador (empregado)(621) & - & - & 2 & 4,4 & - & - & 2 & 4,4 \\
\hline eletrecista (855) & - & - & 2 & 4,4 & - & - & 1 & 4,4 \\
\hline pedreiro (951) & - & - & 1 & 2,2 & - & - & 1 & 2,2 \\
\hline motorista (985) & - & - & - & - & 5 & & 5 & 11,1 \\
\hline serviços gerais (999) & - & - & 1 & 2,2 & 11,1 & & 1 & 2,2 \\
\hline \multirow[t]{2}{*}{ Total } & 29 & & 8 & 17,6 & 8 & & 45 & 100,00 \\
\hline & 64,3 & & & & 17,6 & & & \\
\hline
\end{tabular}

${ }^{\star}$ Classificação Brasileira de Ocupações do Ministério da Saúde (BRASIL. MS, 1987).

Os dados da Tabela 2 mostram a predominância das ocupações leves, com predomínio de aposentados e donas de casa, os quais não são considerados economicamente ativos para a sociedade. Assim, dos 47\% economicamente ativos, as atividades remuneradas mais frequentes no grupo foram as de motorista de caminhão e guarda noturno, ambas classificadas como alteradoras do biorritmo, quer seja quanto ao padrão de sono, quer seja quanto aos hábitos alimentares. 
Ocupações que requerem grande demanda física, trabalhos em turnos e aquelas cuja característica é a monotonia, são fortemente associadas ao infarto. (Bittar, 1992, Kristensen, 1990). Em nosso estudo, 76\% dos economicamente ativos se enquadram nestas modalidades, portanto, considerados de alto risco quanto ao tipo de ocupação desenvolvida.

Outro aspecto importante da relação infarto-ocupação está no nível sócioeconômico que a mesma confere ao trabalhador. A renda familiar de $42,2 \%$ dos pacientes era menor que três salários-mínimos e $28,9 \%$ possuíam renda entre três e seis salários-mínimos, o que demonstra que trabalhadores, ativos ou aposentados, não são adequadamente remunerados pelos seus serviços.

Ao considerarmos que a renda mensal de $71,1 \%$ dos infartados não ultrapassa seis salários-mínimos e que o grau de instrução dos mesmos é precário, estamos diante de um grupo que possui característricas favoráveis ao desenvolvimento de vários tipos de doenças, como o infarto agudo do miocárdio. As causas que favorecem o desenvolvimento de doença isquêmica cardíaca são: prevalência maior de fatores de risco como sedentarismo, tabagismo, obesidade, entre outros; atendimento médico inadequado; dificuldades intelectuais e financeiras para escoIher um estilo de vida mais saudável e meio ambiente mais estressante e desfavorável. (BANCO MUNDIAL, 1991, Bittar, 1992).

Com relação ao estado civil, temos que dos 45 entrevistados, $69 \%$ eram casados; $20 \%$ viúvos; $9 \%$ solteiros e $2 \%$ divorciados. Do total de participantes $91 \%$ referiram ter filhos, sendo que $75,6 \%$ tinham mais de três. Ao associarmos a renda familiar com o número de filhos constatamos que, quanto maior o número de filhos, menor a renda.

Estudos têm demonstrado que o estado civil, muitas vezes, está relacionado com taxas de morbi-mortalidade por doença isquêmica mais elevadas, sendo o coeficiente maior entre os viúvos, solteiros e separados. (Dever, 1988, Koskenvuo, 1980). Outro aspecto é a importância da família, principalmente do cônjuge, no processo de reabilitação do infartado, a qual foi constatada como de fundamental importância para a reintegração social após a doença. (Kellerman, 1993).

Quanto à procedência dos participantes, obtivemos que $84 \%$ deles eram provenientes da cidade de Ribeirão Preto e de outros municípios que compõem esta região administrativa. Tal resultado reafirma a análise feita por Figueiredo(1987), na qual o Hospital das Clínicas é um "pólo" de atração para os pacientes da região quando da necessidade de atendimento de saúde.

\subsection{DADOS RELACIONADOS AO ESTILO DE VIDA}

Buscamos levantar os aspectos apontados por Lalonde(1984) e que, quando presentes no estilo de vida do indivíduo, podem caracterizar os chamados riscos autocriados.

O primeiro dado levantado foi referente aos hábitos alimentares. A dieta é um dos principais fatores do estilo de vida associado ao aparecimento de doença isquêmica cardíaca, uma vez que determina outros fatores de risco como aumento de colesterol total e LDL-colesterol; hipertensão arterial sistêmica; obesidade e 
diabetes mellitus não insulino-dependente. (Guimarães, 1992, Kannel, 1987).

Para analisarmos a dieta dos pacientes utilizamos a descrição feita por Boog; Motta e Bon (1985), que consideram três grandes grupos alimentares, os quais devem estar presentes em uma dieta equilibrada. Esses grupos são: alimentos construtores (fontes de proteínas, cálcio e ferro), reguladores (fontes de vitaminas, sais mineirais e celulose) e energéticos (fornecedores de hidratos de carbono e gorduras).

Com base nas respostas fornecidas pelos pacientes sobre quais eram os alimentos mais freqüentemente consumidos nas principais refeições (almoço e jantar), obtivemos que, do ponto de vista nutricional, a dieta dos pacientes era equilibrada. No almoço, $80 \%$ dos participantes consumiam alimentos dos três grupos alimentares, enquanto que no jantar tal número diminuia para 55,5\%.

Com relação à freqüência do consumo de alimentos de cada grupo, vimos que os entrevistados consumiam maior quantidade de alimentos energéticos (arroz, pães, massas,...) e menor quantidade de reguladores (legumes, verduras e frutas), o que corrabora os estudos de autores como Mondini; Monteiro (1994). Tais autores relataram diminuição na quantidade de fibras consumidas pelas populações ocidentais, substituindo os cereais de alto teor de fibra, como o pão e arroz integrais, por alimentos beneficiados e industrializados; e o aumento no consumo de carnes concomitante à diminuição de hortaliças e frutas.

Em decorrência das dificuldades de se investigar as quantidades dos alimentos consumidos pelos pacientes neste tipo de estudo, optamos por indagar sobre a frequência com que certos alimentos eram ingeridos pelos pacientes. Devido as suas contribuições para o desenvolvimento da ateroesclerose, alimentos de origem animal e ricos em gorduras foram os escolhidos, tais como as carnes vermelhas, ovos, leite e derivados. Embora sejam importantes fontes de proteínas, dependendo da frequência e da quantidade com que são ingeridos, podem tornar-se prejudiciais em decorrência dos altos teores de gorduras em suas composições. O consumo excessivo de alimentos ricos em açúcares, como doces e refrigerantes, e de bebidas alcoólicas, levam ao aumento da obesidade, hipercolesterolemia e diabetes clínico. (Boog; Motta; Bon, 1985, Guimarães, 1992). A dieta apresenta-se como risco autocriado para as doenças cardiovasculares, como o infarto, quando caracterizado por uma super alimentação, alta ingestão de gorduras e carboidratos. (Lalonde, 1974).

O consumo de doces e refrigerantes foi menor que três vezes por semana para $66,6 \%$ dos pacientes, sendo preocupante apenas para aqueles $15,5 \%$ que referiram consumir tais produtos diariamente. 
TABELA 3: Freqüência do consumo de alimentos construtores de importância para a doença isquêmica cardíaca, pelos 45 pacientes infartados.

\begin{tabular}{|lrc|}
\hline Freqüência & $\begin{array}{c}\geq \mathbf{X} \text { /semana } \\
\mathbf{( \% )}\end{array}$ & $\begin{array}{c}\boldsymbol{\Delta} \mathbf{X} / \text { semana } \\
\mathbf{( \% )}\end{array}$ \\
\hline carne bovina gorda & 29,0 & 71,0 \\
carne suína & 8,9 & 91,1 \\
aves com pele & 11,1 & 88,9 \\
ovos & 22,2 & 77,8 \\
leite integral & 55,5 & 44,5 \\
\hline
\end{tabular}

Conforme demonstrado na Tabela 3, nossos dados mostraram que a maior parte do grupo estudado consumia carnes vermelhas e ovos, de acordo com as recomendações da Sociedade Brasileira de Cardiologia (COLESTEROL, 1994), para uma dieta de baixo nível de gordura animal, ou seja até três vezes por semana. O leite foi o alimento que teve sua frequência de consumo maior que três vezes por semana, para $55 \%$ dos entrevistados. Tal produto, embora referido como integral em nossa tabela, inferimos que seja o leite tipo "C", o consumido pelos pacientes, por ser mais barato e coerente com o poder aquisitivo do grupo estudado. A vantagem do leite "C", sob o ponto de vista de ingestão de gorduras é seu baixo teor de lipídeos.

Colombo (1995), encontrou resultados semelhantes em seu estudo e, dosando os níveis de colesterol e triglicerídeos de infartados, encontrou taxas desejáveis de colesterol total e de triglicerídeos, respectivamente, em $52 \%$ e $71 \%$ dos entrevistados.

Quanto às quantidades consumidas, embora não tenha sido objeto de investigação neste estudo, podemos considerar que, diante das condições sócioeconômicas da maioria dos entrevistados e de apenas 31,1\% dos mesmos possuírem algum grau de obesidade, é provável que o consumo desses alimentos seja feito em quantidades não danosas ao organismo.

Outro aspecto investigado foi a ingestão de gorduras pelos pacientes. A ingestão das mesmas através do consumo de alimentos fritos ocorreu para $57,8 \%$ do grupo estudado, com frequência maior ou igual a três vezes por semana. Tal fato pode contribuir para a elevação do rendimento energético da dieta e, quando não acompanhado pelo aumento do gasto energético, pode levar a obesidade. (Mondini; Monteiro,1994).

Quanto à gordura utilizada no preparo das refeições, $71,2 \%$ dos pacientes referiram o uso de óleos de origem vegetal, principalmente o de soja, reconhecidos como os mais saudáveis para o organismo.

Nas últimas décadas, houve a substituição, pela população brasileira, da banha de porco e toucinho por óleos vegetais. No entanto, esta modificação não 
deve ter ocorrido em consequência da conscientização da população quanto aos benefícios que tal troca proporciona à saúde, e sim em decorrência do comportamento de oferta e dos preços mais acessíveis do óleos vegetais (Mondini; Monteiro, 1994).

Diante dos resultados obtidos podemos considerar que, quanto aos hábitos alimentares, o grupo estudado não se apresentou como de risco para o desenvolvimento do infarto.

Outro risco autocriado estaria no uso de bebidas alcoólicas, fumo e medicamentos, no caso das doenças cardiovasculares, os anticoncepcionais hormonais. (Lalonde, 1974).

A maioria dos entrevistados $(66,6 \%)$ referiu não consumir ou consumir raramente bebidas alcoólicas, o que nos permite afirmar que, para tais pessoas, 0 álcool não esteve presente como fator de risco ou risco autocriado para a doença isquêmica cardíaca. O risco para a mesma, relacionado ao consumo de álcool, parece existir apenas para os grandes consumidores (BANCO MUNDIAL, 1991).

Quanto ao tabagismo, temos no cigarro um dos maiores responsáveis por mortes prematuras por cardiopatia, sendo a causa mais importante de mortes por afecções das coronárias (Kannel, 1987, Rosemberg, 1987).

Em nosso estudo, $80 \%$ dos entrevistados eram fumantes e ex-fumantes, sendo a maior porcentagem entre os indivíduos do sexo masculino (Tabela 4). Nossos dados reforçam os da OPS (1992)que apontam o tabagismo como sendo mais prevalente entre os homens do que entre as mulheres, tanto para os fumantes quanto para os ex-fumantes.

TABELA 4: Distribuição dos 45 pacientes, segundo o hábito de fumar e o sexo.

\begin{tabular}{|l|cc|cc|rc|}
\hline Hábito de fumar & \multicolumn{2}{|c|}{ Homens } & \multicolumn{3}{|c|}{ Mulheres } & \multicolumn{2}{c|}{ Total } \\
fumante & $\mathrm{n}$ & $\%$ & $\mathrm{n}$ & $\%$ & $\mathrm{n}$ & $\%$ \\
ex-fumante & 18 & 40,0 & 7 & 15,5 & 25 & 55,5 \\
não fumante & 11 & 24,5 & - & - & 11 & 34,5 \\
\hline Total & 1 & 2,2 & 8 & 17,8 & 9 & 20,0 \\
\hline
\end{tabular}

Os fumantes encontravam-se, predominantemente, na faixa etária dos 50-70 anos. O risco individual de desenvolvimento de doença cardíaca isquêmica é o dobro em fumantes e, na faixa etária de 45-65 anos este risco encontra-se triplicado. Quando estão presentes fatores como tabagismo, hipertensão arterial e hipercolesterolemia, a probabilidade do desenvolvimento da doença torna-se oito vezes maior. (BRASIL, 1993, Rosemberg, 1987). Dos 25 fumantes, 44\% eram hipertensos conhecidos, $8 \%$ referiram hipercolesterolemia e outros $8 \%$ referiram ambas enfermidades. 
O típico fumante de risco é aquele que vem consumindo muitos maços por dia, durante muitos anos, tornou-se tabagista muito jovem e inala a fumaça do cigarro. (Underhill; Stephen, 1984). Tal perfil, parece corresponder a 2/3 dos fumantes do sexo masculino do nosso estudo.

O risco de infarto nos ex-fumantes decresce rapidamente nos primeiros cinco anos. Porém, naqueles que consumiam mais de 20 cigarros por dia, o risco só se iguala ao dos não fumantes após 15 anos de abandono do vício, segundo Rosemberg (1987). No grupo estudado, os 11 (24,5\%) ex-fumantes haviam parado de fumar há mais de cinco anos. Porém, 8,9\% deles fumaram mais de 20 cigarros por dia e tinham deixado de fumar há menos de 15 anos, o que segundo o autor acima, manteria o risco de infarto para os mesmos.

Estudos realizados no Brasil indicaram que o consumo de cigarros é mais provável entre os pobres e os menos educados. (BANCO MUNDIAL, 1991). Tal fenômeno também pode ser observado em nosso estudo.

Quanto ao uso de medicamentos, temos a utilização dos anticoncepcionais orais relacionado à incidência de doenças cardíacas em suas usuárias. Das 15 mulheres que participaram do estudo, apenas $3(20 \%)$ estavam em idade fértil. Destas, uma $(6,7 \%)$ estava fazendo uso de pílulas anticoncepcionais na época do infarto. Tal paciente estava fazendo uso do produto há sete meses, mesmo sendo, tabagista e hipertensa e tendo mais de 35 anos, condições que contra-indicam o uso de tal método contraceptivo,. Outras 4 (26,6\%) pacientes, já no pós-menopausa, relataram uso de hormônios para fins contraceptivos no passado. A duração do uso variou de 1, 4, 13 e 20 anos, respectivamente. Outra infartada fez uso de estrógenos para reposição hormonal no pós-menopausa.

Estudos prospectivos como os do "Royal College of General Practitioners", demonstraram que o nível de mortalidade por doenças circulatórias em usuárias de anticoncepcionais orais era cinco vezes maior que entre as não usuárias. 0 uso de estrógenos por longos períodos, em mulheres acima de 35 anos e fumantes, aumenta o risco de doença cardíaca isquêmica. (Johansson; Vedin, Willrelmsson, 1983). Atualmente, estudos têm demonstrado que os novos anticoncepcionais hormonais, com menores taxas de estrógenos, podem possuir efeitos protetores ao sistema cardiovascular feminino. (Eake, 1993).

Outro risco autocriado relacionado às doenças cardiovasculares é a falta de exercícios físicos e de relaxamento das tensões diárias (Lalonde, 1974). A falta de exercícios físicos regulares, caracterizando o sedentarismo, foi o fator de risco mais prevalente no grupo, atingindo $88,9 \%$ dos entrevistados. As atividades físicas aeróbicas e regulares aumenta a capacidade física do indivíduo e possuem um importante papel na prevenção primária e secundária destas doenças. (Fletcher, 1992).

Um aspecto levantado pelo Ministério da Saúde (BRASIL, 1993) foi que mesmo nas horas de lazer o brasileiro deixou de realizar atividades que exigem esforços físicos como esportes, danças e passeios, restringindo seu lazer às atividades sedentárias e ao ambiente doméstico, centrado em programas de televisão. 
A falta de recreação e de relaxamento das tensões diárias é um risco autocriado, presente no estilo de vida atual e associado com doenças ligadas ao estresse, tais como hipertensão arterial, doença cardíaca isquêmica e úlcera péptica ( Lalonde, 1974).

O estresse é tido como fator de risco possível, mas ainda não claramente estabelecido para tal doença. No entanto, é considerado como fator desencadeante de angina pectoris, infarto do miocárdio e morte súbita. (Kannel, 1987, MancilhaCarvalho, 1992).

O estresse foi por nós investigado através do ambiente referido como facilitador de estresse aos pacientes (55,5\% referiram o ambiente doméstico); alterações no padrão de sono (37,8\% relataram dormirem mal com frequência) e recordação de fatos que tenham trazido preocupação e tristeza na época da ocorrência do infarto $(26,7 \%$ referiram recordar fatos que acreditam ter propiciado ou contribuído com o desencadeamento da doença).

$\mathrm{Na}$ maioria das vezes, o estresse foi traduzido como "tensão nervosa", "nervosismo" e "angústia" pelos entrevistados ao se referirem às tensões do dia-adia doméstico e do ambiente de trabalho, bem como às dificuldades financeiras e desarmonias na convivência com os familiares, foi a causa mais freqüente, citada pelos pacientes, para explicar a ocorrência do infarto.

A relação do estresse com o ambiente social e psicológico negativos, presente nas classes mais baixas, tem sido objeto de estudos. Dever (1988) refere que as mudanças na vida, o estresse e a maneira como as pessoas enfrentam tal estresse são muitas vezes desencadeadores de várias doenças. Como exemplos de reações à luta cotidiana, cita: o tabagismo, a obesidade e o padrão de comportamento tipo $A$, caracterizado por ser agressivo, competitivo e ambicioso, vivendo sob premência de tempo. Enquanto estudos do BANCO MUNDIAL (1991) apontam que os fatores de risco individuais são mais altos entre os pobres e que o fator de risco mais importante, subjacente à doença do adulto no Brasil é a desigualdade do sistema econômico e social.

Diante da caracterização sócio-econômica do grupo estudado e das considerações acima, temos uma situação favorável à geração do estresse para a maioria dos pacientes estudados.

\subsection{DADOS DA ORGANIZAÇÃO DOS SERVIÇOS DE SAÚDE (SISTEMA DE ATENÇÃO À SAÚDE)}

Embora a definição deste elemento, dentro do modelo utilizado, aborde aspectos mais abrangentes da organização dos serviços de atenção à saúde, nossa preocupação se restringiu à caracterização da inserção e seguimento dos pacientes nos serviços por eles procurados, buscando informações com relação ao tratamento de doenças associadas, uso de medicamentos, conhecimento do diagnóstico de infarto e os tipos de informações solicitadas sobre a atual enfermidade.

Dos 45 pacientes, 68,9\% referiram utilizar os serviços de saúde para tratamento de suas doenças crônicas, consideradas como fatores de risco para doença 
isquêmica cardíaca. O uso de medicamentos foi citado pelo mesmo número de infartados. A utilização de outros serviços de saúde, além do Hospital das Clínicas, foi citado por $78 \%$ dos mesmos, principalmente os serviços da rede básica de saúde, para atendimento de emergências e seguimentos clínicos.

Com relação ao conhecimento do diagnóstico de infarto agudo do miocárdio, $3 / 4$ do grupo sabia do motivo da atual internação e, as causas mais freqüentes, apontadas para justificar a ocorrência da doença, foram estresse e tabagismo. Os resultados obtidos nos levam a questionar o tipo de assistência que esses pacientes vêm recebendo, uma vez que a maioria não associava a existência de doenças como a hipertensão arterial, dislipidemias e diabetes, presentes nas histórias clínicas de muitos deles, com a ocorrência do infarto. Embora 55,5\% dos entrevistados tenham referido ser hipertensos, a hipertensão arterial teve $6 \%$ do total de frequência das causas apontadas pelos pacientes.

Quanto ao tipo de informações solicitadas, $74,6 \%$ dos entrevistados desejavam obter conhecimento referente à fisiopatologia, reabilitação, prognóstico e gravidade do infarto. Tal fato demonstra a necessidade dessas pessoas entenderem e se localizarem diante da nova situação.

\section{CONSIDERAÇÕES FINAIS}

Diante das características apresentadas pelo grupo estudado e com base na bibliografia consultada, entendemos que os quatro elementos que compõem o modelo de "Campo de Saúde" apresentaram aspectos que evidenciam suas possíveis contribuições no desencadeamento do infarto.

Assim, no elemento biologia humana tivemos a presença de fatores de risco modificáveis (hipertensão arterial, diabetes e dislipidemias) e não modificáveis (sexo, idade e história familiar positiva para doença cardiovascular); no meio ambiente constatamos um meio social pobre, com indivíduos de baixo nível educacional e com pequena renda familiar mensal; quanto ao estilo de vida, verificamos a presença de riscos autocriados para tabagismo, sedentarismo, falta de recreação e relaxamento das tensões diárias e, no que se refere ao elemento organização dos serviços de saúde vimos que a maioria já estava inserida no sistema de saúde para tratamento de doenças crônicas e de importância para o desenvolvimento da doença cardíaca isquêmica; $3 / 4$ do pacientes tinham conhecimento do diagnóstico atual, mas solicitavam informações referentes à fisiopatologia, prognóstico e recuperação após o infarto.

As informações levantadas pelo presente estudo nos indicam a importância de elaborarmos um programa de reabilitação educacional que respeite as limitações sócio-econômicas, culturais e biológicas dos pacientes infartados. $O$ conteúdo do programa deverá satisfazer às necessidades de informações relatadas pelos mesmos e, ao mesmo tempo, orientá-los para a presença de fatores de risco em suas vidas, uma vez que poucos deles correlacionaram suas histórias clínicas e seus estilos de vida com a ocorrência do infarto. 


\section{REFERÊNCIAS BIBLIOGRÁFICAS}

1. ABURDENE, P.; NAISBITT, J. Megatendências para as mulheres. 2. ed. Rio de Janeiro: Rosa dos Tempos, 1994. Cap. 5, p. 159-198: A megatendência da menopausa.

2. BANCO MUNDIAL. Brasil novo desafio à saúde do adulto.Washington,1991. $134 \mathrm{p}$.

3. BITTAR, O.J.N.V. Retorno ao trabalho após revascularização do miocárdio. Ribeirão Preto, 1992. 84 p. Tese (Doutorado).- Faculdade de Medicina de Ribeirão Preto, Universidade de São Paulo.

4. BOOG, M.C.F.; MOTTA, D.G.; BON, A.M.X. Alimentação natural: prós \& contras. São Paulo: Ibrasa, 1985. Cap.2, p 20-51: O que precisamos saber sobre nutrição.

5. BRASIL. Ministério da Saúde. Secretaria de Assistência à Saúde. Departamento de Programa de Saúde. Coordenação de Doenças Cardiovasculares. Doenças cardiovasculares no Brasil. Sistema Único de Saúde - SUS: dados epidemiológicos; assistência médica. Brasília: MS, 1993. 36 p.

6. BRASIL. Ministério da Saúde. Secretaria Nacional de Ações Básicas de Saúde. Divisão Nacional de Epidemiologia. Classificação brasileira de ocupações para o subsistema de informações sobre mortalidade. Brasília: MS, 1987. 102 p.

7. BRIODY,ME. The role of nurse in modification of cardiac risk factors. Nurs. Clin. North Am., v.19, n.3, p.387-96, 1984.

8. COLESTEROL. Pressão, SBC, v.1, n.1, p.32-8, out.1994.

9. COLOMBO, R.C.R. Estilo de vida e fatores de risco de pacientes com primeiro episódio de infarto agudo do miocárdio. Ribeirão Preto, 1995. 164 p. Dissertação (Mestrado). Escola de Enfermagem de Ribeirão Preto, Universidade de São Paulo.

10. DEVER, G.E.A. A epidemiologia na administração dos serviços de saúde. São Paulo: Pioneira, 1988. 394p.

11. EAKER, E.D. et al. Cardiovascular disease in women. Circulation, v.88, p.19992009, 1993.

12. FIGUEIREDO, A.F. Estudo da demanda regional de hospitalização em Ribeirão Preto, São Paulo, no ano de 1987. Ribeirão Preto, 1994. 64p. Dissertação (Mestrado). Faculdade de Medicina de Ribeirão Preto, Universidade de São Paulo.

13. FLETCHER, G.F. et al. Statement on exercise. Benefits and recommendations for physical activity. Programs for all americans. Circulation, v. 86, n.1, p.3403, 1992.

14. FUNDAÇÃO IBGE. Anuário estatístico do Brasil, 1993. Rio de Janeiro, 1993.

15. GIANINI, S.D. et al. Fatores de risco para coronariopatia. In: SOCIEDADE BRASILEIRA DE CARDIOLOGIA DO ESTADO DESÃOPAULO. Cardiologia: atualização e reciclagem. Rio de Janeiro: Atheneu, 1994. Cap 24, p.260-67. 
16. GYARFAS, I. Lifestyle for preventing cardiovascular disease. J. Cardiovasc. Pharmacol., v.16, p.1-2, 1990. Suplement 8.

17. GUIMARÃES, A.S. Cardiopatia coronária: aspectos epidemiológico e preventivos. Arq. Bras. Cardiol., v.59, n.1, p.5-11, 1992.

18. HENTINEN, M. Teaching and adaptation of patients with myocardial infarction. Int. J. Nurs. Stud., v.23, n.2, p.125-38, 1986.

19. INTERNATIONAL LIPID INFORMATION BUREAU (ILIB) LATINO AMERICA. Recomendaciones de ILIB para el diagnóstico de las dislipidemias en Latinoamérica. Cardiovasc. Risk Factors, v.3, n.1, p.10-27, 1994. Suplemento 1.

20. JOHANSSON, S.; VEDIN, A.; WILHELMSSON, C. Myocardial infarction in women. Epidemiol Reviews, v.5, p.67-95, 1983.

21. KANNEL, W.B.; McGEE, D.; GORDON, T. A general cardiovascular risk profile: the Framingham study. Am. J. Cardiol., v.38, p.46-51, 1976.

22. N_ New perspectives on cardiovascular risk factors. Am. Heart J., v.114, n.1,p.213-9, 1987. Part 2.

23. N Natural history of cardiovascular risk. In: HOLLENBERG, N.K. (ed). Hypertension: mechanisms and therapy. Philadelphia: Ed. Current Medicine, 1995. Cap.5, p.2 - 22.

24. KELLERMAN, J.J. Long-term comprehensive cardiac care: the perspectives and tasks of cardiac reabilitation./ Editorial/ Eur. Heart J., v.14, p.1441-4, 1993.

25. KOSKENVUO, M. et al. Differences in mortality from ischemic heart disease by marital status and social class. J. Chron. Dis., v.33, p.95-106, 1980.

26. KRISTENSEN, T.S.; MANCILHA-CARVALHO, J.J. Ambiente, condições de trabalho e doenças cardiovasculares. Arq. Bras. Cardiol., v.55, n. 4, p.223-6, 1990.

27. LALONDE, M. A new perspective on the health of canadians: a working document. Otawa, 1974. 76p.

28. LAURENTI, R. Epidemiologia das doenças cardiovasculares no Brasil. Arq.Bras. Cardiol., v.38, n.4, p.243-48, 1982.

29. . O declínio das doenças cardiovasculares como causa de morte. I Editorial/. Rev. Saúde Pública, v.20, n.5, p.339-40, 1986.

30. LINDSAY, C.; JENNRICH, J.A.; BIEMOLT, M. Programmed instruction booklet for cardiac rehabilitation teaching. Heart \& Lung, v.20, n.6, p.648-53, 1991.

31. LOLIO, C.A. et al. Tendência da mortalidade por doença isquêmica do coração nas capitais de regiões metropolitanas do Brasil, 1979-89. Arq. Bras. Cardiol., v.64, n.3, p.195-9, 1995.

32. MANCILHA-CARVALHO, J.J. Antecedentes da doença coronária: os fatores de risco. Arq.Bras. Cardiol., v.58, n.4, p.263-7, 1992.

33. MONDINI, L.; MONTEIRO, C.A. Mudanças no padrão de alimentação da população urbana brasileira (1962-1988). Rev. Saúde Pública, v.28, n.6, p.433-9, 1994. 
34. OPS. Tabaco o salud: situación en las Americas: um informe de la Organización Panamericana de la Salud. Washington: Organización Panamericana de la Salud, 1992. $430 \mathrm{p}$.

35. PAFFENBARGER, R.S.; HALE, W.E. Work activity and coronary heart mortality. N.Engl.J. Med., v.292, n.1, p.545-50, 1975.

36. PINNEO, R. Living with coronary artery disease. The nurse's role. Nurs. Clin. North Am., v.19, n.3, p.459-67, 1984.

37. REGO, R.A. et al. Fatores de risco para doenças crônicas não-transmissíveis: inquérito domiciliar no município de São Paulo, SP (Brasil). Metodologia e resultados preliminares. Rev. Saúde Pública, v.24, n.4, p.277-85, 1990.

38. ROSEMBERG, J. Tabagismo e saúde. Informações para profissionais da saúde. Brasília, 1987. 49p.

39. SÃO PAULO (Estado). Hospital das Clínicas de Ribeirão Preto. Relatório de morbidade por patologia: 01/01/93 a 31/12/94. Ribeirão Preto: PRODESP, 1996. 15 p.

40. STOVSKY, B. Nursing interventions for risk factor reduction. Nurs. Clin. North Am., v.27, n.1, p.257-70, 1992.

41. UNDERHILL, S.L.; STEPHEN, S.A. Coronary heart disease: risk factors. In: UNDERHILL, S.L. et al. Cardiac. nursing, 2. ed. Philadelphia: J.B. Lippincont, 1984. Cap.19, p. 194-206.

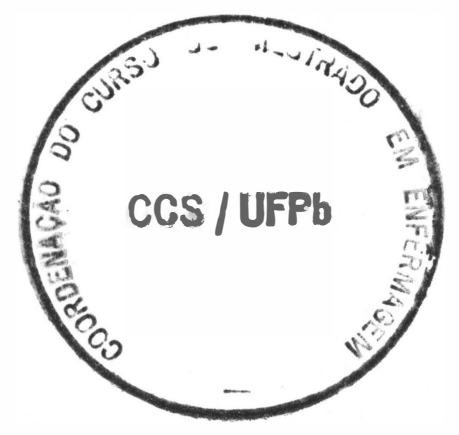

